\title{
Editorial commentary: To DMSA or not, that is the question.
}

More than a decade has gone by since the first papers appeared on cortical imaging of the kidneys using DMSA scanning (to study both acute pyelonephritis and later chronic scarring). Clinical and experimental work has shown its sensitivity in detecting renal inflammation and later evolution of scarring. Yet few papers appear, and these are mainly from only a few centers. Why the lag? What has happened?
Why the resistance to replacing IVPs, VCUs, and ultrasonography in pediatric UTI evaluation with DMSA scanning? The following comprehensive review by Dr. Slovis is worth reading. It provides a fair, detailed study. I asked two colleagues to respond. Dr. John Connor, a pediatric urologist at Babies and Children's Hospital of New York, had actually worked in Dr. Slovis' hospital in his pediatric uro- logic fellowship. Dr. Robert Seigle, also from Babies \& Children's Hospital, is an experienced pediatric nephrologist. Their surgical and medical answers to Dr. Slovis are of interest.

There is, as in all medicine, no one right way. The discussion should keep all of us re-evaluating our standard methods of workup.

W. E. Berdon

\section{Is there a single most appropriate imaging workup of a child with an acute febrile urinary tract infection?}

\section{T.L. Slovis}

Department of Pediatric Imaging, Children's Hospital of Michigan, 3901 Beaubien Boulevard, Detroit, MI 48201-2196, USA

Received: 20 March 1995

Whenever there is uncertainty about a single correct course of action, many options are presented. The less certain the course of action, the more vociferous and sure are the protagonists. The discussion may be "full of sound and fury, signifying nothing" [1]. Rather than get entangled in the politics of modality supremacy or in the ritualistic approach to imaging of the urinary tract, I believe we should start with the following objective: Find the most efficacious imaging procedure for diagnosing pyelonephritis, use this procedure as the initial imaging modality, and give the clinician optimal information to try and prevent the sequelae of pyelonephritis - post-infectious nephropathy (PIN; Table 1) [2,3]. By optimizing the diagnosis of pyelonephritis with minimal necessary imaging and, in the best of worlds, preventing PIN, we are utilizing the most cost-effective approach. Remember, charges are not as relevant as utilization (most patients do not pay full charge ${ }^{1}$ ). When one better test can be substituted for two tests, utilization is decreased.

Talner et al. define pyelonephritis clinically - syndrome of flank pain, tenderness, and fever accompanied by the laboratory findings of bacterial renal infection and pathologically - bacterial infection of kidney with

\footnotetext{
${ }^{1}$ Relative charges at Children's Hospital of Michigan ( 1 = least expense): ultrasonography $=1$, cystography $=1$, nuclear medicine $=$ 2 , computed tomography $=3$.
}

acute inflammation involving both renal pelvis and parenchyma [2]. Most importantly, they state that "some patients whose kidneys fulfill the pathologic criteria for acute pyelonephritis lack clinical or laboratory evidence of the disease" [2].

My reasons for beginning with the definition of pyelonephritis is that the most severe consequences of urinary tract infection (end-stage renal disease secondary to PIN) occur only after pyelonephritis - not after cystitis or pyelitis [4]. The clinical localization of urinary tract infection is difficult, especially in the younger child (less than 3 years of age) [5]. It is now accepted that the intravenous pyelogram (excretory urogram) is insensitive; unfortunately, ultrasound is not much better [7,8] (Table 2). Nuclear medicine with its cortical agents technetium $99^{\mathrm{m}}$ dimercaptosuccinate and glucoheptonate in both animal and human studies have achieved outstanding sensitivity and specificity [9-12]. However, there is a newer methodology (DMSA-SPECT) which may even improve on the rates in Table 2 [13]. CT may offer an even more sensitive way to diagnose pyelonephritis, but there are few experimental or clinical data to support its routine use at this time $[2,14]$. The value of CT is that it gives both anatomical and functional information about the kidney.

Since there is little debate about the fact that the diagnosis of pyelonephritis is our primary objective, does everyone with a febrile urinary tract infection have the 